\title{
A polymer-based interface restores light sensitivity in blind rats
} \author{
Benfenati ${ }^{1}$

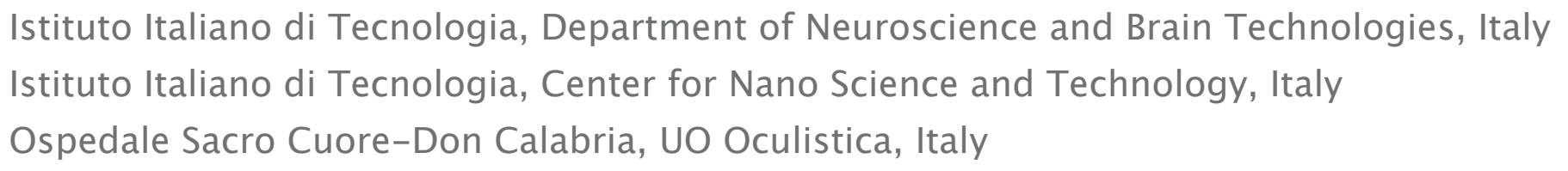

Diego Ghezzi ${ }^{1 *}$, Maria Rosa Antognazza², Maurizio Mete ${ }^{3}$, Grazia Pertile $^{3}$, Guglielmo Lanzani ${ }^{2}$ and Fabio

Progressive degeneration of photoreceptors is one of the major causes of adult blindness in "industrialized" countries; as an example, Retinitis pigmentosa defines a set of monogenic hereditary retinal diseases, with a prevalence of 1:4000 worldwide, caused by single mutations in over 50 genes. Moreover, it is thought that a significant number of implicated genes still await identification (Hartong et al., 2006; Wright et al., 2010;Daiger et al., 2013). In the past 20 years, several treatments have been attempted to treat Retinitis pigmentosa as well as many other retinal genetic diseases, including: drug therapy ( Leveillard and Sahel, 2010), gene therapy (Smith et al., 2012), stem/progenitor cells engrafting (Pearson et al., 2012), optogenetic manipulation (Busskamp et al., 2010), and implantation of prostheses (Weiland et al., 2011;Zrenner et al., 2011). However, despite the enormous efforts and advances in the clinical treatment of eye diseases and some encouraging results in animal models, there is currently no effective cure for the majority of genetic retinal diseases affecting photoreceptors including Retinitis pigmentosa. Sight restoration represents one of the new frontiers for prosthetic devices enabling the electrical stimulation of neurons. In particular, diseases that affect the retinal pigment epithelium and photoreceptors but preserve the inner retinal layers are preferential targets for the implantation of visual prostheses. In our work, we exploited the use of conjugated polymers to generate an organic photovoltaic retinal prosthesis. Our research indicates that organic materials, in particular photovoltaic semiconducting polymers, are suitable for the generation of a fully organic retinal prosthesis, to restore light sensitivity in blindness caused by photoreceptor degeneration.

We discovered that primary neurons can be grown onto a semiconductor polymer layer, without affecting the optoelectronic properties of the photovoltaic material (Antognazza et al., 2009) or the physiological properties of the neuronal network over a long period of time. Moreover, we found that a light pulse is able to depolarize neurons and induce them to fire action potentials (Ghezzi et al., 2011). The spatial resolution of the stimulation paradigm was demonstrated to be in the order of the cell body, in spite of the continuous layer of polymer, indicating that the stimulation is strictly confined to the illuminated area.

This result encouraged us to test the efficacy of photovoltaic polymers in stimulating retinas explanted from albino rats with reproducibly light-induced degeneration of the photoreceptor layer. To this aim, acutely dissected retinas were placed on the organic polymer in a sub-retinal configuration (i.e., external layers in contact with the polymer). Light stimulation of the degenerate retina was observed by monitoring multi-unit activity and field potentials with an extracellular electrode positioned in the retinal ganglion cell layer. Multi-unit activity recordings showed that a light stimulus 16-fold lower than the safe limit for pulsed illumination elicited intense spiking activity in degenerate retinas placed on polymer-coated substrates to levels indistinguishable from those recorded in control retinas. Moreover, to evaluate the efficiency of the interface, a dose-response analysis of spiking activity versus light intensity were performed in degenerate retinas. Spiking activity was observed in degenerate retinas over the polymer with a response threshold below $0.3 \mu \mathrm{W} / \mathrm{mm} 2$, a linear increase in a range corresponding to daylight irradiance, and a response saturation above $100 \mu \mathrm{W} / \mathrm{mm} 2$ (considered the safe limit for chronic illumination). A 4-fold increase in the amplitude of the light response at saturation and a significant left shift of the dose-response curves were obtained in retinas placed over the polymer-coated interface respect to degenerate retinas on glass substrates. Our finding indicates that the interface could mimic functional photoreceptors in activating the processing of the inner retina and is able to rescue normal light sensitivity (Ghezzi et al., 2013).

Following these promising results, we implanted the prosthesis in the eye of rats (Royal College of Surgeons) bearing photoreceptor degeneration due to mutation in the MERTK gene (D'Cruz et al., 2000). These rats are a recognized animal model of human Retinitis pigmentosa. Preliminary experiments performed in a first cohort of animals show that the retina remains well attached over the entire region of the sub- retinal implant without inflammation or fibrosis. Moreover, the ability of the organic prosthesis to restore light sensitivity was first tested by monitoring the pupillary reflex. Pupil constriction in the implanted blind rat was similar to the response of the non-dystrophic animal and significantly higher than the non-implanted blind animal. Finally, visually evoked field potentials were detected in the visual cortex of implanted blind rats, whereas they were undetectable in the non-implanted blind animals. These results open the possibility of developing a new generation of fully organic prosthetic devices for sub-retinal implantation. 

to DG).

\section{References}

Antognazza, M.R., Ghezzi, D., Musitelli, D., Garbugli, M., and Lanzani, G. (2009). A hybrid solid-liquid polymer photodiode for the bioenvironment. Applied Physics Letters 94.

Busskamp, V., Duebel, J., Balya, D., Fradot, M., Viney, T.J., Siegert, S., Groner, A.C., Cabuy, E., Forster, V., Seeliger, M., Biel, M., Humphries, P., Paques, M., Mohand-Said, S., Trono, D., Deisseroth, K., Sahel, J.A., Picaud, S., and Roska, B. (2010). Genetic reactivation of cone photoreceptors restores visual responses in retinitis pigmentosa. Science 329, 413-417.

D'cruz, P.M., Yasumura, D., Weir, J., Matthes, M.T., Abderrahim, H., Lavail, M.M., and Vollrath, D. (200o). Mutation of the receptor tyrosine kinase gene Mertk in the retinal dystrophic RCS rat. Hum Mol Genet 9, 645-651.

Daiger, S.P., Sullivan, L.S., and Bowne, S.J. (2013). Genes and mutations causing retinitis pigmentosa. Clinical genetics 84, $132-141$.

Ghezzi, D., Antognazza, M.R., Dal Maschio, M., Lanzarini, E., Benfenati, F., and Lanzani, G. (2011). A hybrid bioorganic interface for neuronal photoactivation. Nat Commun 2, 166 .

Ghezzi, D., Antognazza, M.R., Maccarone, R., Bellani, S., Lanzarini, E., Martino, N., Mete, M., Pertile, G., Bisti, S., Lanzani, G., and Benfenati, F. (2013). A polymer optoelectronic interface restores light sensitivity in blind rat retinas. Nature Photonics 7, 400-406.

Hartong, D.T., Berson, E.L., and Dryja, T.P. (2006). Retinitis pigmentosa. Lancet 368, 1795-1809.

Leveillard, T., and Sahel, J.A. (2010). Rod-derived cone viability factor for treating blinding diseases: from clinic to redox signaling. Sci Transl Med 2, $26 \mathrm{ps} 16$. Pearson, R.A., Barber, A.C., Rizzi, M., Hippert, C., Xue, T., West, E.L., Duran, Y., Smith, A.J., Chuang, J.Z., Azam, S.A., Luhmann, U.F., Benucci, A., Sung, C.H., Bainbridge, J.W., Carandini, M., Yau, K.W., Sowden, J.C., and Ali, R.R. (2012). Restoration of vision after transplantation of photoreceptors. Nature 485, 99-103. Smith, A.J., Bainbridge, J.W., and Ali, R.R. (2012). Gene supplementation therapy for recessive forms of inherited retinal dystrophies. Gene Ther 19 , $154-161$. Weiland, J.D., Cho, A.K., and Humayun, M.S. (2011). Retinal prostheses: current clinical results and future needs. Ophthalmology 118, $2227-2237$.

Wright, A.F., Chakarova, C.F., Abd El-Aziz, M.M., and Bhattacharya, S.S. (2010). Photoreceptor degeneration: genetic and mechanistic dissection of a complex trait. Nat Rev Genet 11, 273-284.

Zrenner, E., Bartz-Schmidt, K.U., Benav, H., Besch, D., Bruckmann, A., Gabel, V.P., Gekeler, F., Greppmaier, U., Harscher, A., Kibbel, S., Koch, J., Kusnyerik, A., Peters, T., Stingl, K., Sachs, H., Stett, A., Szurman, P., Wilhelm, B., and Wilke, R. (2011). Subretinal electronic chips allow blind patients to read letters and combine them to words. Proc Biol Sci 278, 1489-1497.

Keywords: Conjugated polymers, Photovoltaic materials, Visual Prosthesis, subretinal stimulation, Blindness

Conference: MERIDIAN 30M Workshop, Brixen, Italy, 25 Sep - 25 Sep, 2014. Presentation Type: Oral Presentation Topic: Neuroengineering

Citation: Ghezzi D, Antognazza M, Mete M, Pertile G, Lanzani G and Benfenati F (2014). A polymer-based interface restores light sensitivity in blind rats. Front. Neuroeng. Conference Abstract: MERIDIAN 30M Workshop. doi: 10.3389/conf.fneng.2014.11.00002

Received: 06 Nov 2014; Published Online: 06 Nov 2014.

* Correspondence: Dr. Diego Ghezzi, Istituto Italiano di Tecnologia, Department of Neuroscience and Brain Technologies, Genova, 16163, Italy, diego.ghezzi@me.com 